\title{
LEAK WIDTHS RESULTING FROM PLASMA DIFFUSION IN A MAGNETIC CUSP
}

\author{
R.A. BOSCH and R.M. GILGENBACH \\ Intense Energy Beam Interaction Laboratory, Nuclear Engineering Department, The University of Michigan, \\ Ann Arbor, MI 48109-2104, USA \\ Received 12 January 1988; accepted for publication 16 February 1988 \\ Communicated by R.C. Davidson
}

Several formulas describing cusp leak widths are derived using different assumptions on the natures of the crossfield diffusion and the plasma flow parallel to the magnetic field.

An important property of a quasineutral plasma confined by a magnetic cusp is the leak width - the width of the profile of plasma escaping through the cusp. Recently, Koch and Matthieussent [1] have derived a formula for the leak width of a collisional cusp which differs from the formula used by several other researchers [2-6]. The validity of this new formula has been questioned in an earlier comment [7]. We find that the differing formulas may be derived from a single equation using different assumptions on the natures of the crossfield diffusion and the plasma flow parallel to the magnetic field. This elucidates the regimes under which the different formulas and scaling laws are valid.

The leak width of a quasineutral plasma flowing out of a cusp while undergoing ambipolar diffusion across the magnetic field may be approximated by [4]

$d=\left(D_{\perp} L / v_{\|}\right)^{1 / 2}$,

where $D_{\perp}$ is the coefficient of ambipolar crossfield diffusion, $L$ is the scale length of the cusp, and $v_{\|}$is the speed at which plasma flows out of the cusp.

When the flow of plasma parallel to the magnetic field is noncollisional, then $v_{\|} \approx C_{\mathrm{s}}$, where $C_{\mathrm{s}}$ is the ion acoustic speed $[4,5,8]$. In this case, eq. (1) reduces to

$d=\left(D_{\perp} L / C_{\mathrm{s}}\right)^{1 / 2}$.

This equation has been used by several researchers to describe cusp leak widths [2-6].
For crossfield diffusion resulting from collisions with neutral gas, $D_{\perp}=r_{\mathrm{ce}}^{2} \nu_{\mathrm{e}}$, where $r_{\mathrm{ce}}$ is the electron gyroradius and $\nu_{\mathrm{e}}$ is the electron collision frequency [9]. In this case, eq. (2) results in the scaling

$d \propto P^{1 / 2} B^{-1}$,

where $P$ is the neutral gas pressure and $B$ is the magnetic field strength in the cusp.

If crossfield diffusion is dominated by Bohm diffusion with $D_{\perp}=k T_{\mathrm{e}} / 16 \mathrm{eB}$, then eq. (2) results in the scaling

$d \propto P^{0} B^{-1 / 2}$.

The scaling of eqs. (3) and (4) has been observed experimentally $[4,5]$, with collisional crossfield diffusion dominating over Bohm diffusion at sufficiently high neutral pressures.

On the other hand, if plasma flow parallel to the magnetic field is impeded by plasma-neutral collisions and described by ambipolar diffusion, then $v_{\|}=D_{\|}\left(\mathrm{d} n / \mathrm{d} x_{\|}\right) / n \approx D_{\|} / L$, where $D_{\|}=C_{\mathrm{s}}^{2} / \nu_{\mathrm{i}}$, and $\nu_{\mathrm{i}}$ is the ion collision frequency [9]. In this case, eq.

(1) reduces to

$d=L\left(D_{\perp} / D_{\|}\right)^{1 / 2}$.

When crossfield diffusion results from collisions with a neutral gas, so that $D_{\perp}=r_{\text {cc }}^{2} \nu_{\mathrm{e}}$, then eq. (5) may be written as

$d=L\left(r_{\mathrm{ce}} r_{\mathrm{ci}} / \lambda_{\mathrm{e}} \lambda_{\mathrm{i}}\right)^{1 / 2}$,

where $r_{\mathrm{ce}}$ and $r_{\mathrm{ci}}$ are the electron and ion gyroradii, 
$\lambda_{\mathrm{e}}$ and $\lambda_{\mathrm{i}}$ are the electron and ion mean free paths. Up to a factor of $2 / \pi$, this is the formula of Koch and Matthieussent [1]. The scaling given by eq. (6) is $d \propto P^{1} B^{-1}$.

When crossfield diffusion is dominated by Bohm diffusion while parallel flow results from collisional ambipolar diffusion, eq. (5) results in the scaling

$d \propto P^{1 / 2} B^{-1 / 2}$.

An examination of eqs. (3), (4), (7) and (8) indicates that different scaling laws are obeyed in four regimes, which are differentiated by the nature of the plasma flow parallel to the magnetic field and the type of crossfield diffusion. A leak width scaling with magnetic field as $B^{-1}$ results from collisional crossfield diffusion, while a leak width scaling as $B^{-1 / 2}$ results from Bohm crossfield diffusion. When the ion mean free path is comparable to or larger than the scale length of the cusp, then the flow of plasma parallel to $B$ will not be significantly affected by collisions, so that eqs. (2)-(4) may be applicable. On the other hand, when the ion mean free path is much smaller than the scale length of the cusp, then the flow of plasma parallel to $B$ will be significantly influenced by collisions, so that eqs. (5)-(8) may be more appropriate.
This research was supported by the National Science Foundation under Grant no. ECS-8351837, the Office of Naval Research, and SDIO-IST.

\section{References}

[1] C. Koch and G. Matthieussent, Phys. Fluids 26 (1983) 545.

[2] T.K. Allen, H. Coxell, M. Hill and I.J. Spalding, Proc. APS Topical Conf. on Pulsed high-density plasmas, Report no. LA3770 ( 1967 ) G4;

I.J. Spalding, M.J. Eden, A.D.R. Phelps and T.K. Allen, in: Proc. Third Int. Conf. on Plasma physics and controlled nuclear fusion, Vol. 2 (IAEA, Vienna, 1969) p. 639.

[3] R. Jones, Plasma Phys. 25 (1983) 1535.

[4] R.A. Bosch and R.L. Merlino, Phys. Fluids 29 (1986) 1998.

[5] R.A. Bosch and R.L. Merlino, J. Appl. Phys. 60 (1986) 3056.

[6] R.A. Bosch and R.L. Merlino, Rev. Sci. Instrum. 57 (1986) 2940.

[7] R. Jones, Phys. Fluids 27 (1984) 2780;

G. Matthieussent, Phys. Fluids 27 (1984) 2780.

[8] K.N. Leung, R.E. Kribel, D.G. Fitzsimons and G.R. Taylor, Phys. Lett. A 60 (1977) 203.

[9] F.F. Chen, Introduction to plasma physics and controlled fusion, Vol. 1. Plasma physics (Plenum, New York, 1984) pp. 155-195. 